\title{
Study of Photo-Induced Dichroism in Sudan III Doped in Poly(Methyl Methacrylate) Thin Films
}

\author{
B. $\operatorname{ABBAS}^{a, *}$ AND Y.T. SALMAN ${ }^{b}$ \\ ${ }^{a}$ Atomic Energy Commission, P.O. Box 6091, Damascus, Syria \\ ${ }^{b}$ Physics Department, Faculty of Science, Damascus University, Damascus, Syria \\ (Received December 4, 2013; in final form December 10, 2014)
}

\begin{abstract}
Linear dichroism, dichroic ratio, contrast ratio and order parameters of Sudan III/PMMA guest-host thin films have been investigated with visible polarized laser light. Dichroism increased in an exponential fashion with increase of the pump intensity. Light-induced dichroism and polar order of the dye molecules within the polymer network were reversible. These parameters showed fast increase in their values in an exponential fashion as the pumping process takes place. Also, they decay rapidly in an exponential fashion when the pump light is cut off. Photoisomerisation and polar orientation are dependent on both the molecular structure of the dye and polymer. Two forms of Sudan III molecules (trans and cis) are responsible for the interaction with laser light and forming an anisotropic structure inside the PMMA/Sudan III films. However, there is another set of forms of the dye (keto and $e n o l$ ) has to be considered, which may contribute to dichroism.
\end{abstract}

DOI: $10.12693 /$ APhysPolA.127.780

PACS: 78.20.Ek, 33.55.+b, 78.66.Qn

\section{Introduction}

Photoinduced optical anisotropy in polymer/organic dyes systems is the subject of intensive care and investigations [1]. The importance of these nonlinear optical (NLO) materials for applications are quite obvious in many fields of modern optics [2], among which, optoelectronics, photonics, and optical signal processing. Such materials have been used for polarization holography, optical data storage, integrated optics, all-optical modulation, second-order nonlinear optical (NLO) effects [3], optical recording, optically-controlled optical elements, optical switches [2] and are promising candidates in optical shielding. Azobenzene-based dye molecules form an important set of molecules which have vast interest in research and applications alike. For example, Schab-Balcerzak et al. [4] described the formation of supramolecular azopolymers based on hydrogen bonds as perspective materials for laser induced surface relief gratings (SRGs) and for polarization gratings. They realized supramolecular films on the basis of hydrogen bonds between the functional groups of 4-[4-(3-hydroxypropyloxy)phenylazo]pyridine polymer and azobenzene derivative 4-[4-(6hydroxyhexyloxy)phenylazo]pyridine. Zidan et al. [5] investigated the optical limiting performance of Sudan III dye doped into ethylene propylene dienepolymethylene polymer (EPDM) using $532 \mathrm{~nm}$ pulses from a frequencydoubled Nd:YAG laser. They found that this dye manifests optical limiting effects, with a concentration dependent efficiency. Gayathri and Ramalingam [6] also investigated the third order nonlinear optical property of

*corresponding author; e-mail: pscientific@aec.org.sy
Sudan III in solvents and doped in polymeric films using the Z-scan technique. Multiple diffraction rings were observed when the dye doped in both the liquid and solid media was exposed to a diode-pumped Nd:YAG laser at $532 \mathrm{~nm}$. On the other hand, De Boni and his colleagues [7] studied two-photon absorption (2PA) spectra of Sudan III, Sudan IV and Sudan Red diazoaromatic dyes dissolved in dimethyl sulfoxide (DMSO) using Z-scan technique with fs pulses. They showed that the absence of $2 \mathrm{PA}$ to the $\pi-\pi^{*}$ band is related to the weaker donor/acceptor groups, and lower symmetry of diazoaromatic compounds. Also, the decrease of $2 \mathrm{PA}$ cross-section with temperature is attributed to thermally induced torsions, which decreases the effective conjugation of diazoaromatic molecules. This kind of optical investigations indicated the crucial role of the photoinduced anisotropy in the dye doped systems.

Anisotropy may be induced either by photoinduced isomerization and ordering of the absorbing molecules' dipoles in directions in which light affects them less [2], or by tautomerization where a compound can be represented by two structures that are related by an intramolecular movement of hydrogen from one atom to another [8]. The induced anisotropy manifests itself in dichroism of long-term stability but can be eliminated by either optical or thermal means [9]. Dichroism is a measure of the orientation of the nonlinear optical dye molecules in the host polymer [2]. Dye molecules are well known for their properties of photochromism [10], which is a reversible change between two species having different absorption spectra, which can be induced by photoirradiation. Because photochromism of organic molecules is sensitive to the micro-environment factors such as polarity and free volume, the perturbation of reaction media on photochromic behaviors offers novel information concerning the microstructures of matrices around the 
photoactive molecules [11]. One of the most powerful methods for studying the structure and physical behavior of nonlinear optical guest-host polymeric systems is the measurement of the absorption of linearly polarized light by an ensemble of oriented molecules [12]. To the best of our knowledge, linear dichroism of PMMA/Sudan III thin films has never been studied before. In this work we report results of photoinduced linear dichroism properties of Sudan III molecules that have been incorporated (dissolved) in PMMA polymeric network.

\section{Experimental}

\subsection{Sample preparation}

The starting materials for preparing the guesthost polymeric samples were poly(methymethacrylate) (PMMA, MW: 36000) from Acros Organics, and Sudan III (95\% dye content), from Aldrich. $2 \mathrm{~g}$ of PMMA was dissolved in $20 \mathrm{ml}$ of dichloromethane $\left(\mathrm{C}_{2} \mathrm{H}_{2} \mathrm{Cl}_{2}\right)$. Sudan III was added to the clear dissolved solution of PMMA (equivalent to $5 \%$ of PMMA by weight). The mixture was stirred for $3 \mathrm{~h}$ until the clear solution was obtained with dye molecules fully dissolved. Thin films were dip-coated on transparent glass substrates (film thicknesses of the order $1 \mu \mathrm{m}$ were measured by prism coupling technique and the absorption maximum was at $508.4 \mathrm{~nm})$. Samples were baked in an oven and held at $70^{\circ} \mathrm{C}$ for $1 \mathrm{~h}$ in order to eliminate the residual solvent. Finally, samples were kept in a desiccator at $22^{\circ} \mathrm{C}$ in dark environment.

The samples' best absorption wavelength was found using UV-visible spectrophotometer (Photodiode Array Photospectrometer (PDA) Specord S100, from Analytik Jena) to record the absorption spectrum of PMMA/Sudan III samples.

\subsection{Experimental setup}

Guest-host polymeric thin films become dichroic when pumped with linearly polarized beams from an $\mathrm{Ar}^{+}$laser at $\lambda=514 \mathrm{~nm}$. The probe signal of the beam falling on the sample and subsequently on a photosensor is fed to a personal computer through a low noise current preamplifier (SR570, from Stanford Research Systems), and a DSP lock-in amplifier (SR850, from Stanford Research Systems). An IEEE 488.2 GPIB (from National Instruments) card was used to control and record the experimental data along with a special program written in Borland $\mathrm{C}++$. Figure 1 shows the experimental arrangement of the setup.

Dichroism is measured by a probe beam linearly polarized passing through the sample while it is being subject to the pump beam. The probe beam intensity was kept about $0.03 \%$ of the pump beam intensity. This is an important procedure to use a very weak probe beam with a wavelength identical or very close to the absorption maximum of the dye molecules where any changes in the absorption will be greatest, without influencing the pumping effect of the sample. In this manner, the probe beam absorbance parallel $\left(A_{\|}\right)$and perpendicular $\left(A_{\perp}\right)$

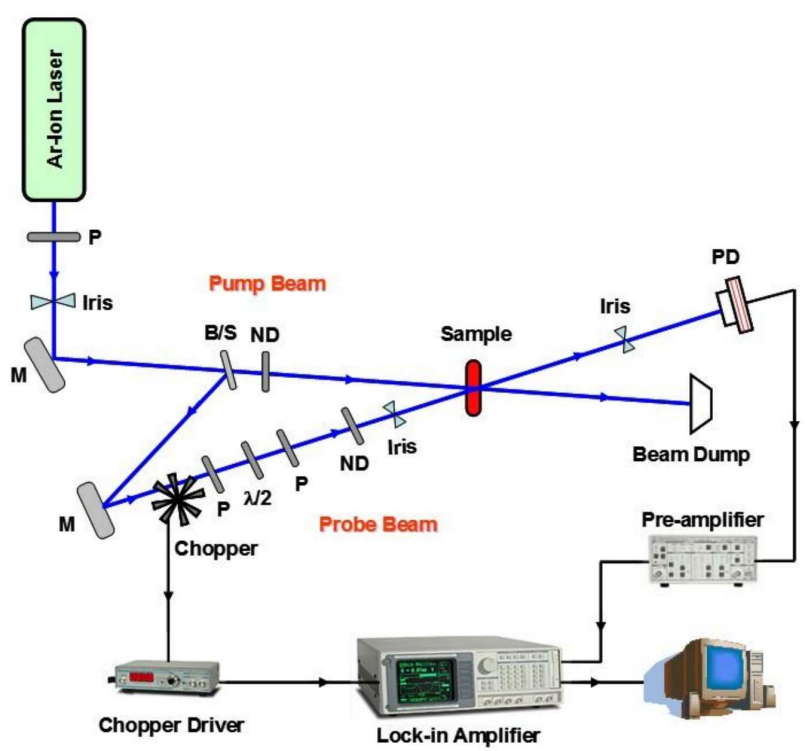

Fig. 1. The experimental setup: $\mathrm{P}-$ polarizer, ND neutral density filter, $\mathrm{B} / \mathrm{S}$ - beam splitter, $\lambda / 2$ - halfwave plate, and $\mathrm{PD}$ - photodiode.

to the electric vector of the pump beam become different. The absorbances were then calculated from [13]:

$$
A_{\|}=-\log \left(\frac{I_{\|}}{I_{0}}\right)
$$

and

$$
A_{\perp}=-\log \left(\frac{I_{\perp}}{I_{0}}\right),
$$

where $I_{0}$ is the intensity of the probe beam when there is no sample, and $I_{\|}$and $I_{\perp}$ are the intensities of the probe beam transmitted through the sample when it is polarized parallel and perpendicular to the pump beam polarization, respectively. The linear dichroism is computed from [2]:

$$
D=\frac{A_{\|}-A_{\perp}}{A_{\|}+2 A_{\perp}} .
$$

\section{Results and discussion}

Figure 2 shows the chemical structure of PMMA polymer (a), and the chemical structure of Sudan III molecule and its tautomeric forms (b). Figure 3 shows that the maximum absorption wavelength for PMMA/Sudan III prepared samples is at $\lambda=508.4 \mathrm{~nm}$. Since it is most suitable for linear dichroism investigations to pump and probe the samples with laser wavelengths identical or close to the maximum absorption of the samples, a wavelength of $\lambda=514 \mathrm{~nm}$ from a multi-line Ar-ion laser (543-MAP-A02, from MellesGriot) was used. A series of experiments was carried out in order to elucidate the influence of linearly polarized light. Samples were pumped (one sample at a time) with several intensity levels, at which the transmitted probe beam intensities were recorded simultaneously. Photoinduced changes in the transmitted probe beam intensities are plotted in Fig. 4. This figure shows the transmission spectra of 
PMMA/Sudan III thin films at different parallel polarized laser intensities of the probe, and depicts the increase in transmitted light intensities with respect to increase in the laser pump intensities.
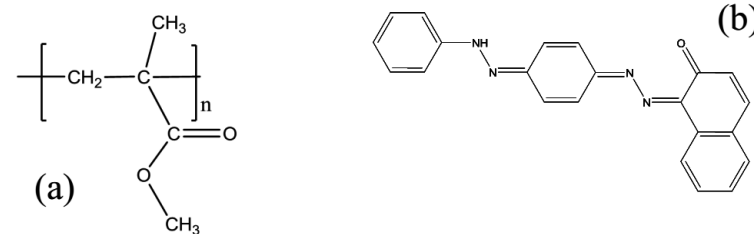

Tautomer 2 (keto form)

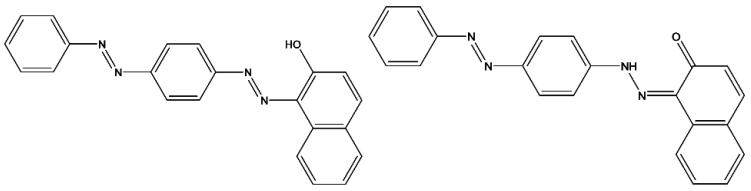

\section{Sudan III (enol form) Tautomer 1 (keto form)}

Fig. 2. The chemical structure of PMMA polymer (a), Sudan III molecule and its tautomeric forms (b).

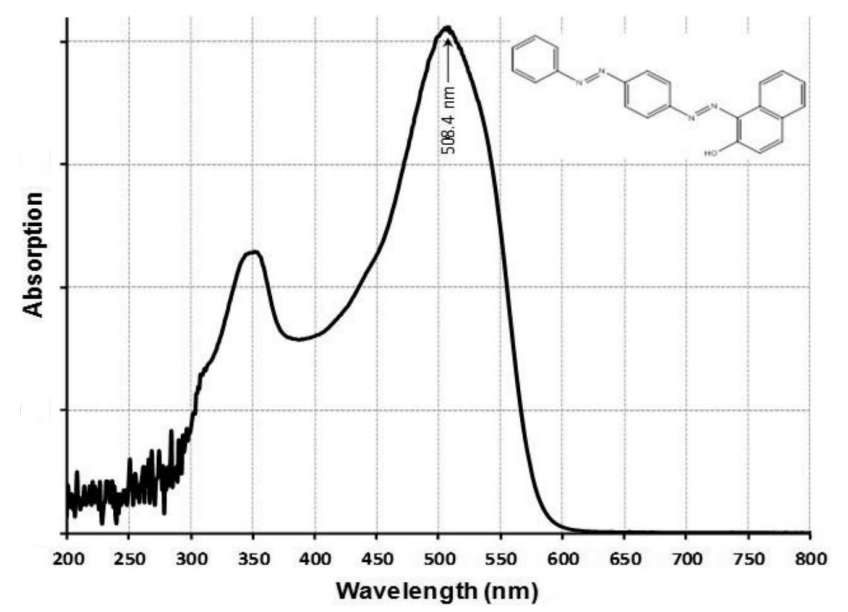

Fig. 3. UV-Visible absorption spectrum of PMMA/Sudan III samples. The chemical structure of Sudan III dye is shown in the inset.

A similar trend was observed for the transmission spectra of PMMA/Sudan III at different perpendicular polarized laser intensities (Fig. 5). Looking at Figs. 4 and 5 one can see that the intensities of the transmitted parallel polarized laser light are greater than those of the transmitted perpendicular polarized laser light by an order of 10. This may be attributed to the contribution the dipole moments of the dye molecules to polar orientation. This contribution is largely affected by the laser light polarization state, in which parallel laser light induces a stronger polar order in the samples in the direction perpendicular to the direction of the pump light polarization state. This leads to a progressive greater increase in the transmitted probe light with parallel polarization. Similarly, a smaller increase in the transmitted probe light

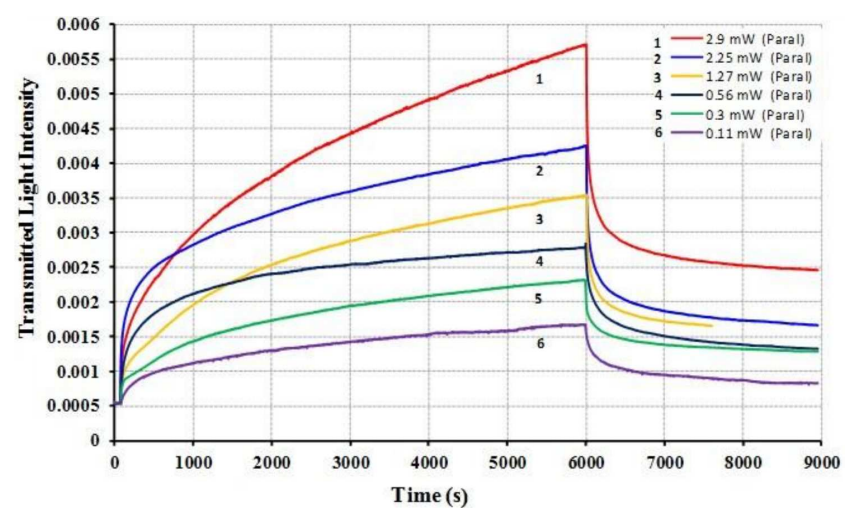

Fig. 4. Transmitted spectra of PMMA/Sudan III at different parallel polarized laser intensities.

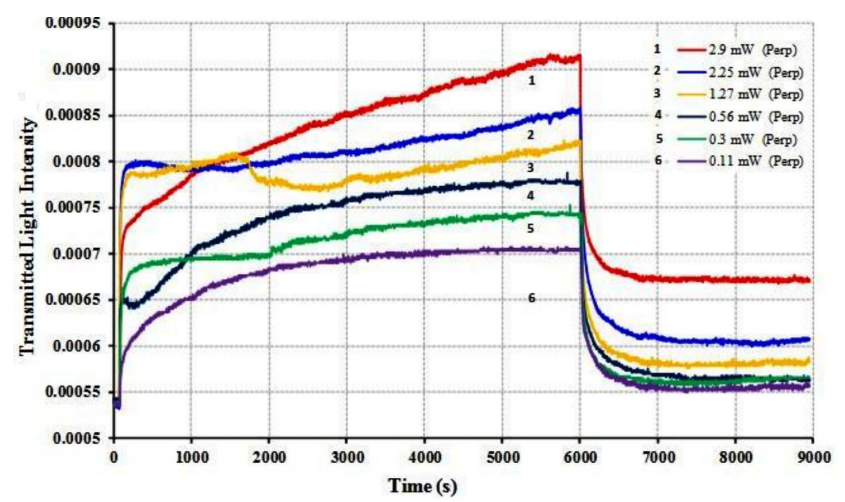

Fig. 5. Transmitted spectra of PMMA/Sudan III at different perpendicular polarized laser intensities.

with perpendicular polarization was also recorded. However, at low pumping intensities, in the case of orthogonal probe and pumping beams, there existed a two-step photostationary state $(0.3-2.25 \mathrm{~mW}$ pump intensities). It is believed that there was a competitive tautomerization process taking place. This process indicates the existence of - at least - two tautomers with different activation energies, so that at low pumping energy only one tautomer carries out the phototautomerization cycle. The other tautomer with higher activation energy starts to participate in the phototautomerization process only when there is sufficient energy to proceed. This will become the case after diminishing the number of the tautomers of the lower activation energy and obtaining the first photostationary state. Then the second phototostationary state prevails as a result of devoting more pumping energy for this part of the two-step photostationary state. At higher pumping intensities, the first part of this phototautomerization state becomes less obvious since there will be enough energy for both tautomers to participate in the phototautomerization process. This interesting phenomenon was not noticed in the case of parallel beam probing. There is now an extensive theoretical research in our laboratory to study the characteristics of possible tautomers, and relate that to the idea of the competition processes that could be responsible for the appearance of the two-step photostationary state. 


\subsection{Linear dichroism}

Linear dichroism of PMMA/Sudan III was calculated using Eq. (3) at different laser light intensities: 0.11, 0.3, $0.56,1.27,2.25$, and $2.9 \mathrm{~mW}$; at $22^{\circ} \mathrm{C}$. Figure 6 depicts the dichroism results, which shows that dichroism increases as the pump intensity does.

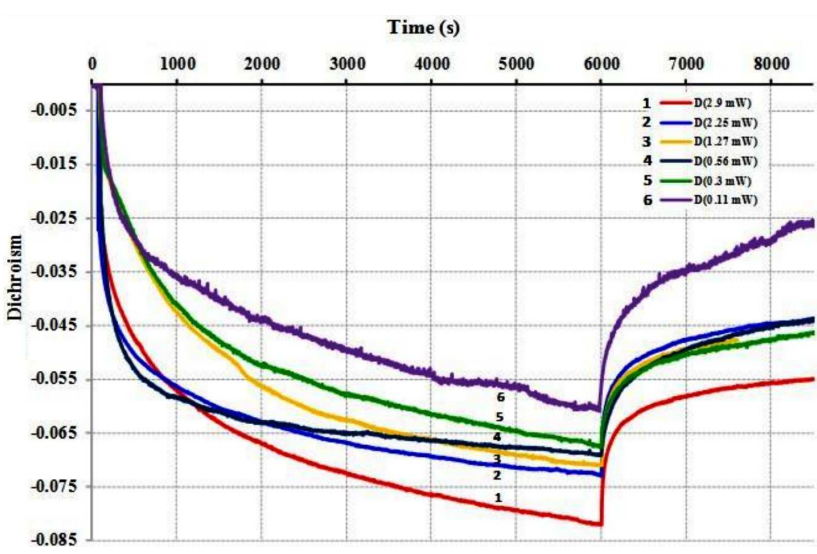

Fig. 6. Linear dichroism of PMMA/Sudan III thin films at different polarized laser light intensities.

\subsection{Dichroic ratio}

The Sudan III dye has a high dichroic ratio because of its rod-like molecular shape. Dichroic ratios $(D R)$ are calculated by [14]:

$$
\begin{aligned}
& D R_{1}=\frac{D_{\mathrm{S}}}{D_{\mathrm{I}}}, \\
& D R_{2}=\frac{D_{\mathrm{S}}}{D_{\mathrm{R}}},
\end{aligned}
$$

where $D_{\mathrm{S}}$ is the dichroism value at the photostationary state, $D_{\mathrm{I}}$ is the dichroism value at the isotropic state, and $D_{\mathrm{R}}$ is the dichroism value after $2000 \mathrm{~s}$ of relaxation.

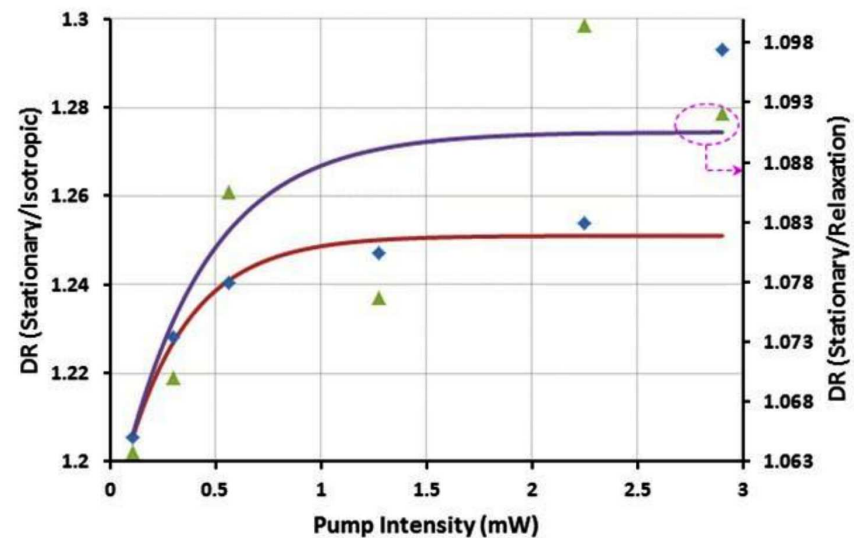

Fig. 7. Dichroic ratio of PMMA/Sudan III thin films at different polarized laser light intensities for both parallel and perpendicular polarization states.

Figure 7 expresses the growth of $D R$ during irradiation of the samples with different pump intensities. This growth follows an exponential logistic function of the form

$$
D R_{1}=A_{0}+A_{1} \mathrm{e}^{-\left(\frac{I}{t}\right)},
$$

where $A_{0}$ is an offset value of $\mathrm{DR}_{1}$ at the absence of the pump light, $A_{1}$ is the dichroic ratio of the sample prior to irradiation, and $t$ is the decay constant. The decay in the polar order within the samples due to cutting-off the pump beam follows an exponential fashion of the form:

$$
D R_{2}=A_{0}+A_{1} \mathrm{e}^{-\left(\frac{I}{t}\right)},
$$

where $A_{0}$ is an offset value of $D R_{2}$ at the absence of the pump light, $A_{1}$ is the dichroic ratio of the sample prior to relaxation, and $t$ is the decay constant.

\subsection{Contrast ratio}

The orientation of the Sudan III dye molecules was controlled using laser light, which enabled the contrast ratio of the Sudan III dye to be obtained by optically switching [15]. Absorption evolution during irradiation with polarized laser light was estimated via Eqs. (1) and (2), as well as after cutting-off irradiation. The ratio between maximum absorption in the on and off states was calculated using [15]:

$$
C R=\frac{A_{\text {off }}}{A_{\text {on }}},
$$

where $A_{\text {off }}$ and $A_{\text {on }}$ are the absorbance at the maximum absorption wavelength at the off- and on-states, respectively. The contrast ratio of the PMMA/Sudan III was calculated from the absorption of the pump laser light at on- and off-states. Figure 8 depicts the contrast ratio of

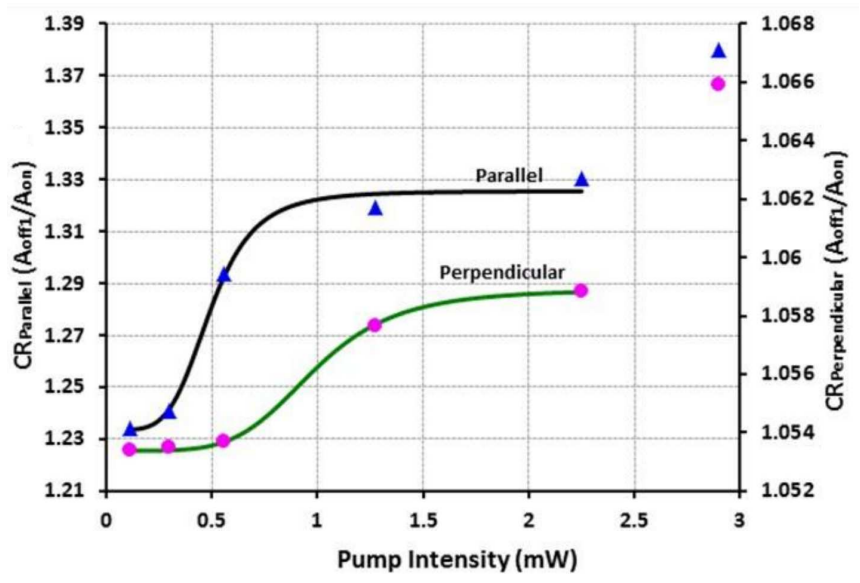

Fig. 8. Contrast ratio of PMMA/Sudan III thin films as a function of pump laser light intensity.

PMMA/Sudan III at different parallel and perpendicular polarized laser intensities. This figure shows a linear increase of the contrast ratio with increase of the laser pump intensities. Further increase in the pump intensity accelerates the growth of the contrast ratio until a stable level is reached. However, the last value of the pump intensity causes the sample to bleach, which leads to deterioration in the absorption characteristics due to the destruction of the dye molecules. This behavior follows an exponential fashion, which can be expressed by a logistic function of the form 


$$
C R=A_{2}+\frac{A_{1}-A_{2}}{1+\left(\frac{I}{I_{0}}\right)^{p}},
$$

where $A_{1}$ and $A_{2}$ are the initial and final values of the contrast ratio, respectively, at which $I$ becomes asymptotic; $I_{0}$ is the center value of the growth section of the contrast ratio at which $I$ acquires a value equal to half of the amplitude of the curve, i.e., the point midway between $A_{1}$ and $A_{2} ; I$ is the pump intensity, and $p$ is the power of this function which controls the steepness of the curve.

\subsection{Order parameter}

The contrast ratio, $\mathrm{CR}$, directly relates to the order parameter for the transition moment, which is dependent on the size, shape, polarity, steric effect and aggregative properties of the dye molecules [15]. The order parameter can be calculated by

$$
S=\frac{D R-1}{D R+2},
$$

where $D R$ is the dichroic ratio [15]. Figure 9 shows an exponential increase of the order parameter in respect of the pump intensity and reaches the maximum value of 0.999 at the pump intensity value of $1.27 \mathrm{~mW}$. However at the

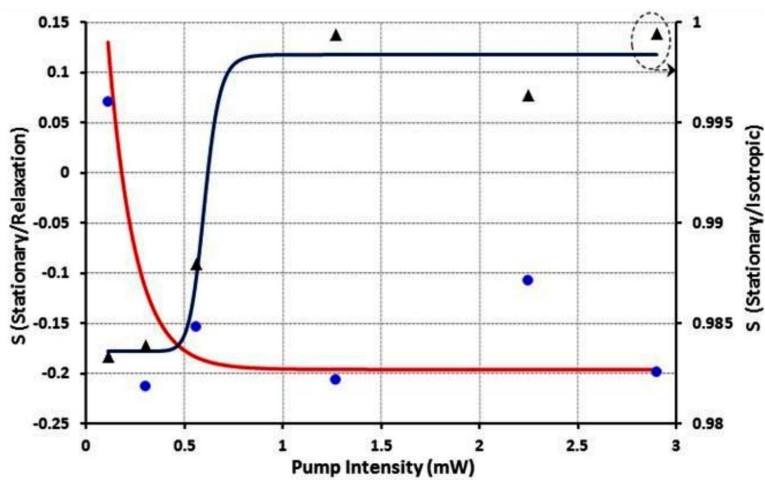

Fig. 9. Order parameter of PMMA/Sudan III thin films at different polarized laser light intensities.

value of $2.9 \mathrm{~mW}$ of the pump intensity bleaching symptoms begin to appear, which lead to a decrease in order parameter due to the destruction process of organic dye molecules. However, the order parameter deteriorates in an exponential fashion as soon as the pump light is cut-off. Moreover, this deterioration is also noticed with respect to the pump intensity levels. The build-up order parameter with respect to the increase in the laser pump intensity can be described via an exponential function of the form

$$
S_{\text {build-up }}=A_{2}+\frac{A_{1}-A_{2}}{1+\left(\frac{I}{I_{0}}\right)^{p}},
$$

where $A_{1}$ and $A_{2}$ are the initial and final values of the order parameter, respectively, at which $I$ becomes asymptotic, $I_{0}$ is the center value of the build-up section of the order parameter, at which $I$ acquires a value equal to half of the amplitude of the curve, i.e., the point midway between $A_{1}$ and $A_{2}, I$ is the pump intensity, and $p$ is the power of this function which controls the steepness of the curve. The decay in the order parameter due to cutting-off the pump light - with respect to the pump intensity - may be described as

$$
S_{\text {relaxation }}=A_{0}+A_{1} \mathrm{e}^{-\left(\frac{I}{t}\right)},
$$

where $A_{0}$ is an offset value of the order parameter at the absence of the pump light, $A_{1}$ is the dichroic ratio of the sample prior to relaxation, and $t$ is the decay constant.

The realized dichroic ratio, contrast ratio, and order parameter are comparable with some published works in literature. For example: Ahmad et al. [16] studied a nematic liquid crystal host polymer doped with $\mathrm{Su}-$ dan black B and investigated using UV-Vis polarized spectroscopy. The achieved dichroic ratio was 1.336 , and the order parameter value was 0.1 at a saturation poling voltage of $40 \mathrm{~V}$. As such, the accomplished order parameter can be improved by the aid of DC poling fields. Ghanadzadeh et al. [15] reported the results of the electro-optical behaviour of Sudan black B, Sudan III, and Sudan IV solutions in nematic solvents using polarized spectroscopy in a guest-host system. They used an electric field (30 V saturated voltage) in order to control the orientation of the dye molecules, which enabled the contrast ratio of the dye to be obtained by electrical switching. They obtained a contrast ratio of 3.7, 3.4, and 5.7 for Sudan III, Sudan IV, and Sudan black B, respectively. Moreover, the order parameters were $0.47,0.43$, and 0.61 for Sudan III, Sudan IV, and Sudan black B, respectively. Yoon et al. [17] have synthesized a DR19-containing polymer (PEA) and a DANS diamine-containing polymer (PAS). Using the corona poling technique with several $\mathrm{kV}$ of poling field, they estimated order parameters to be 0.35 and 0.16 for PEA and PAS, respectively. This kind of research emphasized on employing high poling DC voltage in order to achieve higher order and better anisotropic structures, but in our present work we have not. Instead, the anisotropy in the thin film sample was due to optical pumping only.

\section{Mechanisms of photoinduced ordering}

Sudan III dyes are very efficient photochromic anisotropic molecules. Basically, each molecule has two isomeric shapes, trans (the more stable one) and cis (Fig. 10), which describe the orientational ordering of photoisomerizable Sudan III dye molecules in PMMA polymer. The delocalization of conjugated $\pi$-electrons along the principal axis of the Sudan III dye molecules makes their polarizability strongly anisotropic $\left(A_{\|} \gg\right.$ $\left.A_{\perp}\right)$. However, the molecular anisotropy of the dye molecules plays an important role as well, in which the anisotropy of the trans form can lead to a bias in its angular distribution. This occurs during pumping with polarized light so that the probability for trans molecules to be pumped into the cis state upon irradiation is proportional to the angle between the long axis of the cis molecule and the beam polarization direction [18]. The orientation of the trans form is defined using polar coordinations by the 
angles $\theta$ and $\varphi$ (Fig. 11). The probability, $P$, for a trans form molecules to be pumped into the cis state is then given by

$$
P \propto \cos ^{2} \theta \sin ^{2} \varphi
$$

The absorption probability, proportional to $\cos ^{2} \theta$, leads to a selective depletion of the trans molecules parallel to the pump light polarization direction and resulting in having more cis molecules. This generates a "hole" in the angular distribution of the trans molecules along the pump beam polarisation direction [19]. Molecules lying in the $x$-direction will be pumped preferentially to the cis state. This leads to having the trans population biased towards the $y$-direction. Cis molecules have weaker absorption, so that a probe beam polarised in the $x$-direction ( $A_{x}$ or $A_{\|}$, parallel to that of the pump beam) does not suffer from absorption as it travels through this sample. Moreover, a probe beam polarized in the $y$ direction $\left(A_{y}\right.$ or $A_{\perp}$, perpendicular to the direction of that of the pump beam) will experience a little reduction in absorption. It is important to notice that angular hole burning leads to a decrease in the absorption in all directions for wavelengths close to the absorption maximum of trans forms. Pump light intensity, and the lifetime of the cis form plays an important role in the extension and the effectiveness of the angular hole burning. For example, if the lifetime of the cis form is very long or the pump intensity is very high, then eventually most trans forms would be pumped into the cis state.

Another model has also to be considered, which is called angular redistribution (AR) model. This model was widely used by Russian researchers [19] to describe

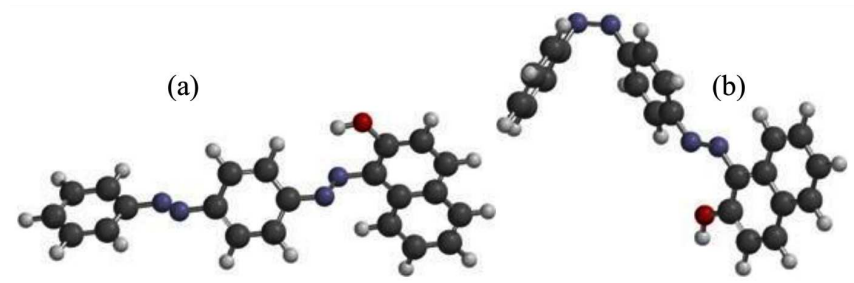

Fig. 10. trans (a) and cis (b) isomers of Sudan III molecules.

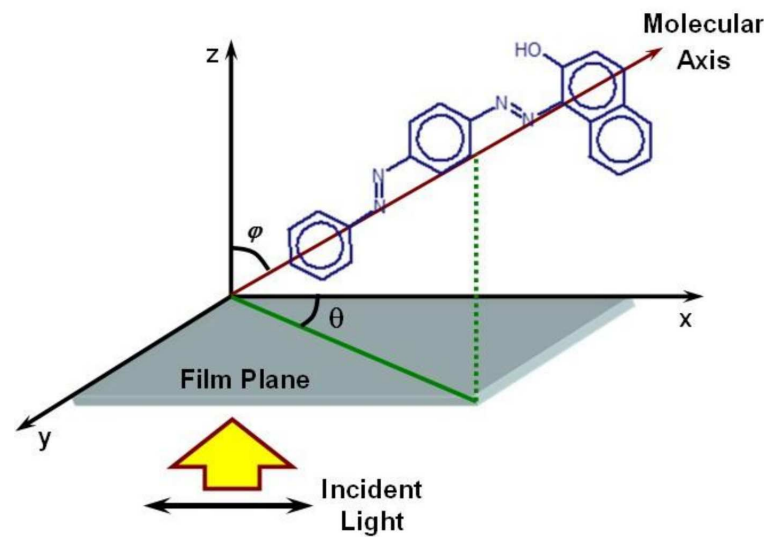

Fig. 11. Dye molecule orientation with respect to the polarization direction of the incident light. how films become birefringent upon irradiation with light. In this model, the relaxation time is very short so that there is no depletion in the population of trans molecules. In other words, the trans molecules are selectively pumped into the cis state according to the $\cos ^{2} \theta$ angular dependence of Eq. (13) and after a very short time they relax back to the trans state either thermally or through photoexcitation with a new orientation [20]. However, if the final trans molecule, after the photoisomerisation cycle, still lies along the polarization direction of the pump light, it will go through a trans-cis-transprocess again. On the other hand, if this trans molecule has another orientation, it is less likely to go through the trans-cis-trans process. As a result of this continuous angular selective interaction, more trans molecules are gradually aligned in a plane perpendicular to the polarization direction of the pump light. This causes the absorption $\left(A_{\|}\right)$parallel to the polarisation direction of the pump light to decrease due to molecules being pumped away from this direction. On the other hand, the absorption $\left(A_{\perp}\right)$ perpendicular to the polarisation direction of the pump light increases as a consequence of more molecules being aligned in this direction. This is manifested in weaker intensity of the transmitted probe light in the case of orthogonal probe-pump beams scheme. Nevertheless, the depletion of the trans molecules away from the polarization direction of the pump beam does not go to completion because of the thermal energy of the trans molecules. Random motion - due to thermal energy - leads to oppose the alignment process [18]. Switching the pump beam off leads to have the anisotropy relaxing due to angular diffusion of the molecules [21].

Figures 4 and 5 can be explained according to the angular hole burning (AHB) model as the trans molecules are being depleted selectively and this process is dominant in the first part of the curves where the increase in the transmitted probe intensity is fast. In the second part, as the angular redistribution takes the advantage, the transmission of the transmitted probe intensity increases progressively. Switching the pump light off causes both transmissions (parallel and perpendicular probes) to decrease suddenly due to fast relaxation of the cis form; but the dichroism originating in the molecular orientation has much larger relaxation time.

Moreover, some researchers mentioned a third mechanism called molecular environment (ME), which is determinant for the stability of the photoinduced order, since it either permits or does not the thermal diffusion in the trans state, when the pump is off. It is also determinant for the kinetics of angular distribution induced by isomerization. The collective effects modify deeply the dynamics of rotation and the competition between optical ordering and other anisotropic effects (such as surface effects) leads to more complicated structures [10].

These models are quite illustrative, and offer a good explanation to the experimental results as long as the photoisomerization process is taken into account only. 
However, such models do not take into account the effect of tautomerization processes occurring during irradiation [22-24]. There is no clear picture of the effect of tautomerization on the light-dye reaction. This effect will be the subject of a separate research in our laboratory to shed the light on its impact on the photo-alignment and dichroism amplitude.

On a different note, some researchers reported that intramolecular hydrogen bonding within the polymer/dye structure plays a role in the photoisomerization process. Ya et al. [25] investigated the effect of intermolecular hydrogen bonding between the hydroxyl groups in the azobenzenechromophores and the carbonyl group in the PMMA polymer. They found that azobenzenes may be anchoring on the PMMA chain, where their rotational motion would be restricted by the free volume in the PMMA. This effect limits the cis-trans back isomerization. However, Ya et al. did not take into account the effect of tautomerization on the photoisomerization process. Nevertheless, the effect of possible hydrogen bonding between the hydroxyl groups in the azobenzenes and the carbonyl group in the PMMA polymer remains an interesting point to look at in our guest-host polymeric system, which will be studied in detail in the future.

\section{Conclusion}

Sudan III dye in PMMA thin film has been studied and showed nonlinear properties. Dichroic properties have been investigated and characterized. PMMA/Sudan III guest-host systems showed difference in the absorption characteristics when probed with parallel and perpendicular polarized laser light in respect of the pump light polarization. Moreover, the absorption increases as the pump intensity does, and a bleaching effect was noticed at pumping intensities larger than $2.9 \mathrm{~mW}$. Dichroism increased in the samples with increase in the pump intensity. All figures showed a rapid increase in the dichroism level at the beginning of the pumping process followed by a slower increase. A saturation state was reached so that no further increase in the dichroism was noticed. Cutting-off the pump light led to a fast deterioration in the dichroism level followed by a slow decrease which takes a very long time to reach the isotropic state of the sample. The dichroic ratio and order parameter showed a fast increase in their values in an exponential fashion as the pumping process taking place. In addition, the dichroic ratio and order parameter decay rapidly in an exponential fashion as well as soon as the pump light is cut off. At $2.25 \mathrm{~mW}$ of pumping intensity, a maximal value of 1.25 of dichroic ratio in the stationary state compared to its value at the isotropic state prior to irradiation was obtained. Also, a maximal value of 1.33 and 1.06 of the constant ratio in parallel and orthogonal configurations, respectively, was recorded. In addition, a maximal value of 0.999 of order parameter was obtained. Such information is valuable in the field of nonlinear optics and applications and highlights the possibility of using such systems in industry applications such as fast optical systems and optical filters, etc.

\section{Acknowledgments}

The authors would like to express their thanks to the Director General of AECS Prof. I. Othman for his continuous encouragement, guidance and support.

\section{References}

[1] M. Ivanov, L. Nikolova, T. Todorov, N. Tomova, V. Dragostinova, Opt. Quantum Electron. 26, 1013 (1994).

[2] B. Abbas, M. Alshikh Khalil, Acta Phys. Pol. A 117, 904 (2010).

[3] Z. Sekkat, J. Wood, E.F. Aust, W. Knoll, W. Volksen, R.D. Miller, J. Opt. Soc. Am. B 13, 1713 (1996).

[4] E. Schab-Balcerzak, A. Sobolewska, J. Stumpe, L. Hamryszak, P. Bujak, Opt. Mater. 35, 155 (2012).

[5] M.D. Zidan, A.W. Allaf, Z. Ajji, A. Allahham, Opt. Laser Technol. 42, 531 (2010).

[6] C. Gayathri, A. Ramalingam, Spectrochim. Acta A 69, 96 (2008).

[7] L. De Boni, A.A. Andrade, S.B. Yamaki, L. Misoguti, S.C. Zilio, T.D.Z. Atvars, C.R. Mendonca, Chem. Phys. Lett. 463, 360 (2008).

[8] Y.C. Martin, J. Comput.-Aided Mol. Des. 23, 693 (2009).

[9] D. Brown, A. Natansohn, P. Rochon, Macromolecules 28, 6116 (1995).

[10] M. Dumont, Mol. Cryst. Liq. Cryst. 282, 437 (1996).

[11] M. Ueda, H.-B. Kim, T. Ikeda, K. Ichimura, J. NonCryst. Solids 163, 125 (1993).

[12] K. Yamaoka, E. Charney, J. Am. Chem. Soc. 94, 8963 (1972)

[13] D.R. Worrall, S.L. Williams, in: Encyclopedia of Modern Optics, Ed. B.D. Guenther, Vol. 1, Academic Press, Amsterdam 2004, p. 31.

[14] A.M. Sarzhevskiı̌, V.A. Gaŭsenok, Anisotropy of Absorption and Luminescence of Polyatomic Molecules, University Press, Minsk 1986 (in Russian).

[15] A. Ghanadzadeh, M.S. Zakerhamidi, H. Tajalli, J. Mol. Liq. 109, 143 (2004).

[16] F. Ahmad, M. Jamil, Y.J. Jeon, L.J. Woo, J.E. Jung, J.E. Jang, Bull. Mater. Sci. 35, 221 (2012).

[17] K.R. Yoon, B.G. So, S.M. Lee, J. Photosci. 12, 95 (2005).

[18] B. Abbas, Ph.D. Thesis, University of Reading, Reading (UK) 1999, p. 100.

[19] M. Dumont, Z. Sekkat, SPIE Proc. 1774, 188 (1992).

[20] T. Todorov, L. Nikolova, N. Tomova, Appl. Opt. 23, 4309 (1984).

[21] Z. Sekkat, M. Dumont, Synth. Met. 54, 373 (1993).

[22] B. Abbas, M. Alshikh Khalil, Acta Phys. Pol. A 115, 857 (2009).

[23] R.A. Sayle, J. Comput. Aided Mol. Des. 24, 485 (2010).

[24] A. Ahmedova, S.P. Simeonov, V.B. Kurteva, L. Antonov, Chem. Central J. 7, 29 (2013).

[25] Q. Ya, X.-Z. Dong, W.-Q. Chen, X.-M. Duan, Dyes Pigments 79, 159 (2008). 